\title{
The Li-F-H Ternary System at High Pressures
}

\author{
Tiange Bi, ${ }^{1}$ Andrew Shamp, ${ }^{1}$ Tyson Terpstra, ${ }^{1}$ Russell J. Hemley, ${ }^{2}$ a) and Eva Zurek ${ }^{1, \text { b) }}$ \\ ${ }^{1)}$ Department of Chemistry, State University of New York at Buffalo, Buffalo, NY 14260-3000, \\ USA \\ ${ }^{2)}$ Departments of Physics and Chemistry, University of Illinois at Chicago, Chicago, Illinois 60607, \\ USA
}

(Dated: 1 January 2021)

Evolutionary crystal structure prediction searches have been employed to explore the ternary Li-F-H system at $300 \mathrm{GPa}$. Metastable phases were uncovered within the static lattice approximation, with $\mathrm{LiF}_{3} \mathrm{H}_{2}, \mathrm{LiF}_{2} \mathrm{H}, \mathrm{Li}_{3} \mathrm{~F}_{4} \mathrm{H}, \mathrm{LiF}_{4} \mathrm{H}_{4}$, $\mathrm{Li}_{2} \mathrm{~F}_{3} \mathrm{H}$ and $\mathrm{LiF}_{3} \mathrm{H}$ lying within $50 \mathrm{meV} /$ atom of the $0 \mathrm{~K}$ convex hull. All of these phases contain $\mathrm{H}_{n} \mathrm{~F}_{n+1}^{-}(n=1,2)$ anions, and $\mathrm{Li}^{+}$cations. Other structural motifs such as $\mathrm{LiF}$ slabs, $\mathrm{H}_{3}^{+}$molecules and $\mathrm{F}^{\delta-}$ ions are present in some of the low enthalpy Li-F-H structures. The bonding within the $\mathrm{H}_{n} \mathrm{~F}_{n+1}^{-}$molecules, which may be bent or linear, symmetric or asymmetric, is analyzed. The five phases closest to the hull are insulators, while $\mathrm{LiF}_{3} \mathrm{H}$ is metallic and predicted to have a vanishingly small superconducting critical temperature. This study lays the foundation for future investigations of the role of temperature and anharmonicity on the stability and properties of compounds and alloys in the Li-F-H ternary system.

\section{INTRODUCTION}

The incorporation of hydrogen in materials is of both fundamental and technological interest. Hydrogen undergoes compound formation with elements across the periodic table, and can adopt various bonding schemes varying from ionic to covalent, to van der Waals when present in molecular form. Technologically, hydrides have been employed in nuclear reactor applications, but their formation can precipitate corrosion and embrittlement ${ }^{1}$. More recently, a wide variety of hydrides have been considered for energy applications such as for hydrogen storage ${ }^{2}$ and in battery materials. ${ }^{3}$

High pressure has been explored as a route to increase the amount of hydrogen that may be stored in materials, ${ }^{4,5}$ and to synthesize novel superconductors. ${ }^{6}$ A broad range of structures can form under pressure, many of which can be very hydrogen rich. Hydrogenic motifs that have been identified include quasimolecular $\mathrm{H}_{2}$ units, atomic $\mathrm{H}^{-}$, as well as extended hydrogenic lattices. ${ }^{7-9}$ Many of these are very hightemperature superconductors, as first established for $\mathrm{H}_{3} \mathrm{~S}$ and $\mathrm{LaH}_{10}$. These phases were predicted ${ }^{10-12}$ and synthesized ${ }^{13,14}$ under pressure, with measured superconducting critical temperatures, $T_{c} \mathrm{~s}$, as high as $203 \mathrm{~K}^{13}$ at $150 \mathrm{GPa}$, and 250$260 \mathrm{~K}$ around $200 \mathrm{GPa},{ }^{15,16}$ respectively. The theoretical studies of compressed binary hydrides have paved the way to interest in more complex ternary systems including $\mathrm{LiPH}_{6}{ }^{17}$ $\mathrm{LiP}_{2} \mathrm{H}_{14},{ }^{18} \mathrm{Li}_{2} \mathrm{MgH}_{16},{ }^{19}, \mathrm{CH}_{4}$ intercalated $\mathrm{H}_{3} \mathrm{~S},{ }^{20,21}, \mathrm{SH}_{3}-$ $\mathrm{SeH}_{3},{ }^{22} \mathrm{MgCH}_{4},{ }^{23} \mathrm{H}_{3} \mathrm{P}_{0.15} \mathrm{~S}_{0.85},{ }^{24} \mathrm{CaSH}_{3},{ }^{25}$ and $\mathrm{CaYH}_{12}{ }^{26}$, which are predicted to possess $T_{c}$ values of $100-473 \mathrm{~K}$ at pressures ranging from 100-230 GPa. In fact, recently a $T_{c}$ of $288 \mathrm{~K}$ has been reported experimentally in a phase (or phases) in the C-S-H ternary system near $270 \mathrm{GPa}^{27}$

The ionic solid $\mathrm{LiF}$ has been employed as a radiation dosimeter, in molten salt coolants for nuclear reactors, and it is a test system for models of ionic solids. With its large

\footnotetext{
a)Electronic mail: rhemley@uic.edu

b)Electronic mail: ezurek@buffalo.edu
}

band gap, $\mathrm{LiF}$ has the lowest refractive index of all common infrared materials. It also possesses the highest UV transmission of any material, being able to transmit significantly into the VUV region. The properties of $\mathrm{LiF}$ render it important for a wide range of applications in high pressure research, and its properties under static high pressures have been widely studied (see Ref. 28 and 29). This includes the use of LiF as a pressure standard and pressure-transmitting medium in diamond anvil cell experiments. ${ }^{30,31}$ Moreover, because LiF is observed to remain optically transparent to at least $900 \mathrm{GPa}^{32}$ it has been employed extensively as a window material in dynamic compression experiments, ${ }^{33}$ e.g. for interferometric velocimetry (VISAR) diagnostics. Because of the importance of $\mathrm{LiF}$ in these experiments, its high pressure properties continue to be of great current interest. ${ }^{28,29,32,34-45}$

The interaction of LiF with hydrogen under pressure has not been explored in detail experimentally or theoretically. Early on it was suggested that $\mathrm{LiH}_{2} \mathrm{~F}$, where a hydrogen atom or $\mathrm{H}_{2}$ molecule is placed in the pseudo-octahedral holes in the $B 1$ structure of $\mathrm{LiF}$, might be a way to achieve the metallization of hydrogen and concomitant superconductivity as discussed above. ${ }^{46}$ Also, possible pressure-induced chemical reactions between hydrogen and $\mathrm{LiF}$ windows in dynamic compression experiments ${ }^{44,45}$ could affect the interpretation of the results. Given the above, and the propensity for formation of stable and metastable hydrides under pressure, we were motivated to apply crystal structure prediction techniques to the $\mathrm{Li}-\mathrm{F}-\mathrm{H}$ ternary system to multimegabar pressure. By now the phase diagrams of the elemental $\left(\mathrm{Li},{ }^{47} \mathrm{H}_{2},{ }^{48}\right.$ and $\left.\mathrm{F}_{2}{ }^{49}\right)$ and binary $\left(\mathrm{LiH}_{n},{ }^{50,51} \mathrm{H}_{n} \mathrm{~F}^{52,53}\right.$ and $\left.\mathrm{LiF}^{40,41}\right)$ systems have been studied computationally at $300 \mathrm{GPa}$, providing the basis for us to explore the high pressure phases of the ternary system.

Using evolutionary crystal structure prediction techniques, we find a number of metastable phases that are within $50 \mathrm{meV} /$ atom of the convex hull and are therefore potentially synthesizable. Common structural motifs present in these phases include $\mathrm{H}_{n} \mathrm{~F}_{n+1}^{-}$anions of various lengths and $\mathrm{Li}^{+}$ counter-cations. Most of these crystalline lattices are calculated to be wide-gap insulators, with the exception of $\mathrm{LiF}_{3} \mathrm{H}$, 
which is predicted to be metallic and superconducting below $0.1 \mathrm{~K}$.

\section{COMPUTATIONAL DETAILS}

Crystal structure prediction searches were performed using density functional theory (DFT) coupled with the evolutionary algorithm (EA), XTALOPT, release 9. ${ }^{54-56}$ Duplicate structures were detected via the XTALCOMP algorithm ${ }^{57}$. EA runs were carried out on $\mathrm{LiFH}_{n}(n=2,3), \mathrm{LiF}_{2} \mathrm{H}_{n}(n=1-2)$, $\mathrm{LiF}_{3} \mathrm{H}_{n}(n=1-3), \mathrm{LiF}_{4} \mathrm{H}_{n}(n=1,4), \mathrm{Li}_{2} \mathrm{~F}_{m} \mathrm{H}(m=1-3)$, $\mathrm{Li}_{2} \mathrm{FH}_{2}, \mathrm{Li}_{3} \mathrm{~F}_{m} \mathrm{H}(m=1-4), \mathrm{Li}_{4} \mathrm{FH}_{4}, \mathrm{Li}_{4} \mathrm{~F}_{3} \mathrm{H}$, and $\mathrm{Li}_{4} \mathrm{~F}_{4} \mathrm{H}$ stoichiometries at $300 \mathrm{GPa}$ employing 2-3 formula units (FU) within the simulation cells. The lowest enthalpy structures obtained from each search were relaxed at $300 \mathrm{GPa}$.

Geometry optimizations and electronic structure calculations were performed using DFT as implemented in the Vienna Ab-Initio Simulation Package (VASP) version 5.4.1, ${ }^{58}$ with the gradient-corrected exchange and correlation functional of Perdew-Burke-Ernzerhof (PBE) ${ }^{59}$ The projector augmented wave (PAW) method ${ }^{60}$ was used to treat the core states, along with a plane-wave basis set with an energy cutoff of $700 \mathrm{eV}$. The $\mathrm{F} 2 s^{2} 2 p^{5}, \mathrm{H} 1 s^{1}$ and $\mathrm{Li} 1 s^{2} 2 s^{1}$ electrons were treated explicitly using the PAW-PBE F, PAW-PBE $\mathrm{H}$ and PAW-PBE Li_sv POTCARs. In the EA searches only the $\mathrm{Li} 2 s^{1}$ electrons were considered as valence, because exploratory EA runs for $\mathrm{LiF}_{4} \mathrm{H}_{3}$ with the two sets of POTCARs found the same lowest enthalpy structures, and their volumes differed by less than $0.5 \%$. The $k$-point grids were generated using the $\Gamma$-centered Monkhorst-Pack scheme, and the number of divisions along each reciprocal lattice vector was chosen such that the product of this number with the real lattice constant was $30 \AA$ in the structure searches, and $50 \AA$ otherwise. The crystal orbital Hamiltonian populations (COHPs), ${ }^{61}$ and the negative of the COHPs integrated to the Fermi level (-iCOHPs) were calculated using the LOBSTER package ${ }^{62}$.

Phonon calculations were performed using VASP combined with the Phonopy ${ }^{63}$ package under the harmonic approximation. The supercells were chosen such that the number of atoms within them was always greater than 100 . Infrared (IR) spectra were simulated using the PhonopySpectroscopy package. ${ }^{64,65}$ Density functional perturbation theory, DFPT, as implemented in the Quantum Espresso $(\mathrm{QE})^{66}$ program, was used to obtain the dynamical matrix of the $\mathrm{LiF}_{3} \mathrm{H}$ phase. The $\mathrm{Li}, \mathrm{F}$, and $\mathrm{H}$ pseudopotentials, obtained from the GRBV pseudopotential library, adopted used the $1 s^{2} 2 s^{0.55}, 2 s^{2} 2^{5}$, and $1 s^{1}$ valence configurations, respectively. The plane-wave basis set cutoff energies were set to $90 \mathrm{Ry}$, and the Brillouin-zone sampling scheme of Methfessel-Paxton ${ }^{67}$ using a smearing of 0.002 Ry and a 12 $\times 12 \times 12 k$-point grid was employed. The electron phonon coupling (EPC), $\lambda$, was calculated using a set of Gaussian broadenings of $0.012 \mathrm{Ry}$, and a $3 \times 3 \times 3$-grid.

The geometry optimization of the $(\mathrm{F}-\mathrm{H}-\mathrm{F})^{-}$anion in the gas phase was performed using the Amsterdam Density Functional (ADF) software package. ${ }^{68,69}$ The basis functions consisted of an all electron triple- $\zeta$ Slater-type basis set with po- larization functions (TZP) from the ADF basis set library, ${ }^{70}$ and the $\mathrm{PBE}^{59}$ functional was employed.

\section{RESULTS}

\section{A. Thermodynamic Stability}

At $300 \mathrm{GPa}$ and $0 \mathrm{~K}$, the thermodynamically stable phases located on the three-dimensional convex hull are the elemental and binary phases: $\mathrm{Li}, \mathrm{H}_{2}, \mathrm{~F}_{2}$, $\mathrm{LiF}, \mathrm{HF}, \mathrm{LiH}, \mathrm{LiH}_{2}$, and $\mathrm{LiH}_{6}$. However, all of the ternary Li-F-H phases uncovered here are dynamically stable. Among them $\mathrm{LiF}_{3} \mathrm{H}_{2}, \mathrm{LiF}_{2} \mathrm{H}, \mathrm{Li}_{3} \mathrm{~F}_{4} \mathrm{H}$, $\mathrm{LiF}_{4} \mathrm{H}_{4}$, and $\mathrm{Li}_{2} \mathrm{~F}_{3} \mathrm{H}$ are within $41 \mathrm{meV} /$ atom of the hull (Figure S3, Table S1), while $\mathrm{LiF}_{3} \mathrm{H}_{3}, \mathrm{Li}_{3} \mathrm{FH}, \mathrm{LiF}_{3} \mathrm{H}, \mathrm{Li}_{4} \mathrm{~F}_{3} \mathrm{H}$, $\mathrm{Li}_{4} \mathrm{FH}_{4}, \mathrm{LiF}_{4} \mathrm{H}, \mathrm{Li}_{2} \mathrm{FH}, \mathrm{Li}_{2} \mathrm{~F}_{2} \mathrm{H}$, and $\mathrm{Li}_{3} \mathrm{~F}_{2} \mathrm{H}$ are within 58$91 \mathrm{meV} /$ atom of the hull. All other phases are less stable with the most unfavorable candidate that was identified, $\mathrm{LiFH}_{3}$, lying $222.5 \mathrm{meV} /$ atom above the hull. As described in Figure $\mathrm{S} 5$, the $\mathrm{LiH}_{2} \mathrm{~F}$ phase found in the EA searches was considerably more stable than those proposed previously by Gilman. ${ }^{46}$

When the zero-point-energy (ZPE) was taken into consideration, $\mathrm{LiF}_{3} \mathrm{H}$ joined the aforementioned five phases to lie less than $50 \mathrm{meV} /$ atom from the hull (Figure S4, Table S2). The reaction enthalpies, $\Delta H_{F}$, of the decomposition of $\mathrm{LiF}_{3} \mathrm{H}_{2}$, $\mathrm{LiF}_{2} \mathrm{H}, \mathrm{Li}_{3} \mathrm{~F}_{4} \mathrm{H}$, and $\mathrm{Li}_{2} \mathrm{~F}_{3} \mathrm{H}$ into $\mathrm{LiF}$ and $\mathrm{HF}$ were computed to be $-13.3(10.9),-29.3(-28.5),-33.1(-33.8)$, and -40.5 (41.1) $\mathrm{meV} /$ atom when the ZPE was neglected (taken into consideration), consistent with the fact that they lie above the convex hull. Other reactions that thermodynamically destabilize two additional phases of interest and their computed reaction enthalpies are: $\mathrm{LiF}_{4} \mathrm{H}_{4} \rightarrow \mathrm{LiF}+3 \mathrm{HF}+\frac{1}{2} \mathrm{H}_{2}\left(\Delta H_{F}=-33.4\right.$ (-37.0) meV/atom), and $\mathrm{LiF}_{3} \mathrm{H} \rightarrow \mathrm{LiF}+\mathrm{HF}+\frac{1}{2} \mathrm{~F}_{2}\left(\Delta H_{F}=\right.$ $-62.5(-49.0) \mathrm{meV} /$ atom).

A recent data-mining study found that the $90^{\text {th }}$ percentile of the $0 \mathrm{~K}$ DFT-calculated metastability of all of the compounds within the Inorganic Crystal Structure Database (ICSD) was $\sim 70 \mathrm{meV} /$ atom $^{71}$. For matter under pressure the choice of the synthetic pathway, including the precursors and temperatures employed, might be able to produce kinetically stable phases. Several examples of metastable hydrides that have been synthesized under pressure include $\mathrm{C}_{x} \mathrm{~S}_{y} \mathrm{H}_{z},{ }^{27,72,73}$ $\mathrm{PH}_{n},{ }^{74-77}$ and $\mathrm{CaH}_{2.5} \cdot{ }^{78}$ Moreover, anharmonic and quantum nuclear effects, not considered here, can be important in determining the stability of compounds, in particular those containing light elements. ${ }^{79}$ Therefore, we chose to analyze the structure, bonding and properties of phases that were within $50 \mathrm{meV} /$ atom of the convex hull at $0 \mathrm{~K}$.

\section{B. Structural Properties}

Our first-principles based crystal structure prediction searches predicted that $P \overline{1} \mathrm{LiF}_{3} \mathrm{H}_{2}$, shown in Figure 1(a), is the closest phase to the $300 \mathrm{GPa}$ convex hull. A prominent structural motif within $\mathrm{LiF}_{3} \mathrm{H}_{2}$ is the V-shaped (FH-F-HF) ${ }^{-}$ anion, whose $\mathrm{H}-\mathrm{F}_{\text {middle }}-\mathrm{H}$ bond angle measures $110.0^{\circ}$, and 
(a)

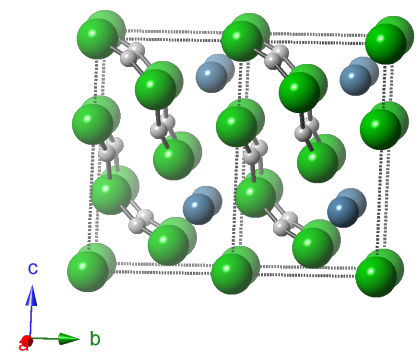

$P \overline{1} \mathrm{LiF}_{3} \mathrm{H}_{2}$

(d)

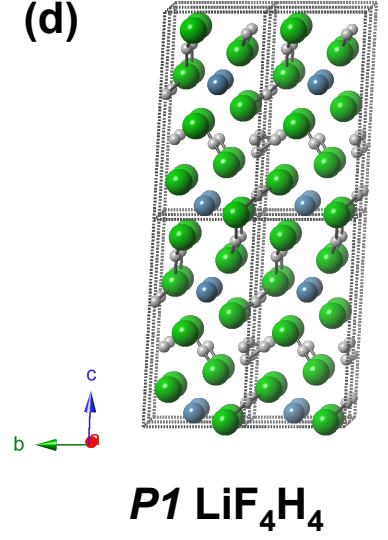

(b)

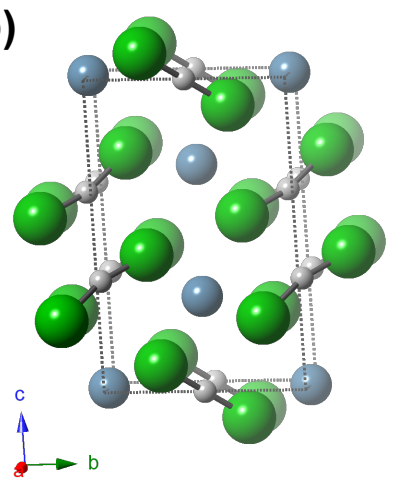

$P \overline{1} \mathrm{LiF}_{2} \mathrm{H}$

(e)

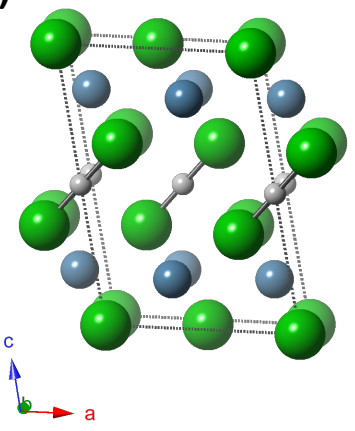

$C 2 / m \mathrm{Li}_{2} \mathrm{~F}_{3} \mathrm{H}$ (c)

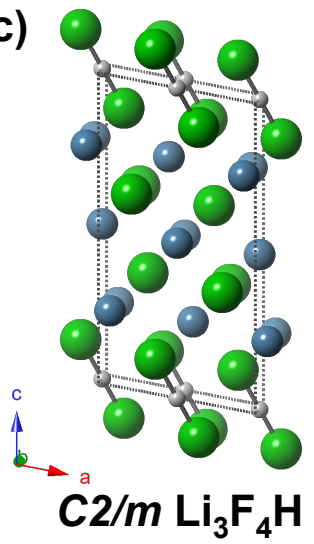

(f)

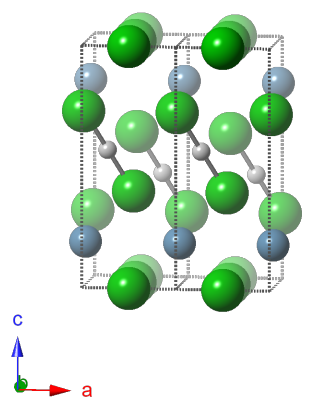

$P 2_{1} / m \mathrm{LiF}_{3} \mathrm{H}$

FIG. 1. Crystal structures of predicted Li-F-H phases at $300 \mathrm{GPa}$ (a) $P \overline{1} \mathrm{LiF}_{3} \mathrm{H}_{2}$, (b) $P \overline{1} \mathrm{LiF}_{2} \mathrm{H}$, (c) $C 2 / m \mathrm{Li}_{3} \mathrm{~F}_{4} \mathrm{H}$, (d) $P 1 \mathrm{Li}_{4} \mathrm{H}_{4}$, (e) $C 2 / m$ $\mathrm{Li}_{2} \mathrm{~F}_{3} \mathrm{H}$, and (f) $P 2_{1} / m \mathrm{LiF}_{3} \mathrm{H}$. Li/F/H atoms are colored blue/green/white.

$\mathrm{F}_{\text {terminal }}-\mathrm{H}-\mathrm{F}_{\text {middle }}$ bond angles measure $160.3^{\circ}$ and $165.7^{\circ}$. The $\mathrm{F}_{\text {terminal }}-\mathrm{H}$ bonds comprising this building block are shorter than the $\mathrm{H}-\mathrm{F}_{\text {middle }}(0.998$ and $1.004 \AA$ vs. 1.107 and $1.099 \AA$, respectively). Each lithium cation is coordinated to six fluorine atoms at distances ranging from 1.587-1.638 $\AA$, and each fluorine atom is coordinated to two lithium cations. One of the Li-F $\mathrm{F}_{\text {terminal }}$ contacts in each (FH-F-HF) ${ }^{-}$unit measures $1.587 \AA$, and the fluorine involved in this contact forms the shortest $\mathrm{H}-\mathrm{F}_{\text {terminal }}$ bond with the largest $\mathrm{F}_{\text {terminal }}-\mathrm{H}-\mathrm{F}_{\text {middle }}$ bond angle.

The phase that is second closest to the convex hull, $P \overline{1}$ $\mathrm{LiF}_{2} \mathrm{H}$, shown in Figure 1(b), resembles $\mathrm{LiF}_{3} \mathrm{H}_{2}$, but the $(\mathrm{FH}-$ F-HF $)^{-}$units are replaced by bifluoride, $(\mathrm{F}-\mathrm{H}-\mathrm{F})^{-}$, anions. This phase is characterized by layers containing $\mathrm{Li}^{+}$and $(\mathrm{F}-$ $\mathrm{H}-\mathrm{F})^{-}$units that are stacked in an $\mathrm{ABCABC}$... fashion along the $c$-axis. The (F-H-F) ${ }^{-}$molecules in layers $A$ and $B$ are bent with a bond angle of $165.3^{\circ}$, and bond lengths of 1.034 and $1.067 \AA$. In going from one layer to the next, the orientation of the $(\mathrm{F}-\mathrm{H}-\mathrm{F})^{-}$units change via a rotation of $180^{\circ}$ around the $c$ axis. In layer $\mathrm{C}$, the $(\mathrm{F}-\mathrm{H}-\mathrm{F})^{-}$anion is linear and symmetric, with both of the H-F bonds measuring $1.037 \AA$.

The gas phase bifluoride anion possesses $D_{\infty h}$ symmetry and is characterized by a strong three-center four-electron bond $(3 \mathrm{c}-4 \mathrm{e}) .{ }^{80}$ In crystals the anion is often bent with two unequal F-H bond lengths, and in some cases may better be described as $\left(\mathrm{F}^{-}\right)(\mathrm{HF})$ with one formal $\mathrm{H}-\mathrm{F}$ two-center twoelectron $(2 \mathrm{c}-2 \mathrm{e})$ bond and one $\mathrm{F}^{-} \cdots \mathrm{H}$ hydrogen bond. ${ }^{81,82}$ A deformation from the ideal symmetry is often a result of the interaction with Lewis acids in the crystal lattice, or with external hydrogen bonds. Although less common, an $(\mathrm{FHF})^{-} \cdot$ HF structural motif, which may be treated as an $\mathrm{F}_{3} \mathrm{H}_{2}^{-}$ion when the bonds are nearly symmetric has also been observed in various salts. ${ }^{83-85}$ A few inorganic compounds containing other suprahalide anions with the general formula $\mathrm{H}_{n} \mathrm{~F}_{n+1}^{-}$and $n=3-6$ are also known. ${ }^{82,86}$

The two molecular motifs present within $P \overline{1} \mathrm{LiF}_{3} \mathrm{H}_{2}$ and $P \overline{1} \mathrm{LiF}_{3} \mathrm{H}_{2}$, (FH-F-HF) ${ }^{-}$and $(\mathrm{F}-\mathrm{H}-\mathrm{F})^{-}$, are also found in the other four compounds that are calculated to lie within $50 \mathrm{meV} /$ atom of the $300 \mathrm{GPa}$ convex hull (Figures 1(c-f)). Bifluoride anions are present in all of the remaining structures, and $\mathrm{LiF}_{4} \mathrm{H}_{4}$ also contains the longer five-atom chain. The smaller $\mathrm{H}_{n} \mathrm{~F}_{n+1}^{-}$anion is the only molecular species found in phases where the $\mathrm{H}: \mathrm{Li}$ ratio $\leq 1$ and the $\mathrm{H}: \mathrm{F}$ ratio $\leq 0.5$.

We now examine the structural peculiarity of these phases, starting with those that only contain the bifluoride anion. The $C 2 / m$ symmetry $\mathrm{Li}_{3} \mathrm{~F}_{4} \mathrm{H}$ phase consists of layers of bifluoride anions that lie parallel to each other stacked along the $c$ axis. These are separated by a five atom thick LiF slab made 
from alternating $\mathrm{Li}$ and $\mathrm{F}$ layers, with Li comprising both the top and bottom layers. At ambient conditions LiF crystallizes in the $B 1(F m \overline{3} m)$ structure, and first-principles calculations have shown that it does not undergo a phase transition to the $B 2$ structure to at least $1 \mathrm{TPa}$ at $0 \mathrm{~K} .{ }^{40}$ Not surprisingly, the $\mathrm{LiF}$ layer in $\mathrm{Li}_{3} \mathrm{~F}_{4} \mathrm{H}$ can be described as a slightly distorted slab cut out from the $B 1$ phase. The segregation into distinct layers observed within $\mathrm{Li}_{3} \mathrm{~F}_{4} \mathrm{H}$ is consistent with the finding that its decomposition into $\mathrm{LiF}$ and $\mathrm{HF}$ is preferred.

The slightly less stable $C 2 / m$ symmetry $\mathrm{Li}_{2} \mathrm{~F}_{3} \mathrm{H}$ phase can be constructed from $\mathrm{Li}_{3} \mathrm{~F}_{4} \mathrm{H}$ by removing two layers (one of lithium and one of fluorine) from the LiF slab. In both of these compounds the (F-H-F) ${ }^{-}$units are linear and symmetric with $\mathrm{H}-\mathrm{F}$ bond lengths of $1.041 \AA$, and the fluorine atom is coordinated to three lithium ions in the LiF slab. $P 2_{1} / m$ $\mathrm{LiF}_{3} \mathrm{H}$ also contains layers of linear $(\mathrm{F}-\mathrm{H}-\mathrm{F})^{-}$anions, however they are not symmetric with $\mathrm{H}-\mathrm{F}$ bond lengths of 1.079 and $1.014 \AA$, and each one of the fluorine atoms within them are coordinated to two lithium ions in the outer most layer of the LiF slab. It is conceivable that phases with these same stoichiometries, but different numbers of atoms in the unit cell, or different stoichiometries that are composed of a single layer of bifluoride anions separated by $\mathrm{LiF}$ layers of varying thickness may also be metastable, with enthalpies of formation falling within the realm of synthesizability.

The most complex structure identified, $P 1 \mathrm{LiF}_{4} \mathrm{H}_{4}$, contains both the short $(\mathrm{F}-\mathrm{H}-\mathrm{F})^{-}$and the long $(\mathrm{FH}-\mathrm{F}-\mathrm{HF})^{-}$anions, along with triangular $\mathrm{H}_{3}^{+}$cations. This compound can also be viewed as a layered phase, with bifluoride anions and $\mathrm{H}_{3}^{+}$cations comprising one set of layers. Each one of the fluorine atoms in these $(\mathrm{F}-\mathrm{H}-\mathrm{F})^{-}$units is coordinated to a single lithium ion within the next layer, which also contains (FH-F$\mathrm{HF})^{-}$motifs wherein both of the terminal fluorines are coordinated to two lithium ions. Thus, each $\mathrm{Li}^{+}$is coordinated to seven fluorines, three belonging to $(\mathrm{F}-\mathrm{H}-\mathrm{F})^{-}$and four to

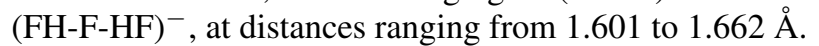

The bifluoride anion linkages in $\mathrm{LiF}_{4} \mathrm{H}_{4}$ are not linear, with a bond angle of $175.5^{\circ}$, nor are they symmetric, with bond lengths measuring 1.035 and $1.042 \AA$. The $\mathrm{H}_{3}^{+}$molecular units resemble the trihydrogen cation - one of the most abundant interstellar molecules ${ }^{87}$, which assumes an equilateral triangle and forms a three-center two-electron (3c-2e) bond. However, the $\mathrm{H}_{3}^{+}$molecule found here is not quite a perfect equilateral triangle, with bond angles of 58.4, 60.4 and $61.2^{\circ}$, and bond lengths of $0.805,0.782$, and $0.799 \AA$. This structural motif has been predicted to exist in $\mathrm{H}_{5} \mathrm{Cl}$, ${ }^{88-90}$ as well as $\mathrm{H}_{2} \mathrm{~F}, \mathrm{H}_{3} \mathrm{~F}$ and $\mathrm{H}_{5} \mathrm{~F}^{90}$ phases under pressure. The geometric parameters of the (FH-F-HF) ${ }^{-}$molecules resemble those in the $\mathrm{LiF}_{3} \mathrm{H}_{2}$ phase, with bond angles of $107.9^{\circ}\left(\mathrm{H}-\mathrm{F}_{\text {middle }}-\mathrm{H}\right)$, as well as $160.1^{\circ}$ and $166.4^{\circ}\left(\mathrm{F}_{\text {terminal }}-\mathrm{H}-\mathrm{F}_{\text {middle }}\right)$, and bond lengths of 0.997 and $1.002 \AA\left(\mathrm{H}-\mathrm{F}_{\text {terminal }}\right)$, as well as 1.110 and $1.098 \AA$ $\left(\mathrm{H}-\mathrm{F}_{\text {middle }}\right)$. Similar to what was found in $P \overline{1} \mathrm{LiF}_{3} \mathrm{H}_{2}$, the terminal fluorine atom that is closest to a lithium ion has the smaller F-H-F bond angle, and the shorter H-F bond length.

\section{Bonding}

Because of the difficulties inherent in measuring the position of hydrogen atoms in crystals, the geometry of the bifluoride anion in simple salts such as $\mathrm{KHF}_{2}{ }^{91}$ and $\mathrm{NaHF}_{2}{ }^{92}$ has been debated. Two types of bonding scenarios are possible: a delocalized $3 \mathrm{c}-4 \mathrm{e}$ bond in the symmetric structure, vs. one $2 \mathrm{c}-2 \mathrm{e}$ bond and one extremely strong hydrogen bond in the unsymmetric geometry. In more complex salts hydrogen bonding with other moieties, or electrostatic interactions with strong Lewis acids can lead to a deviation from linearity. ${ }^{81}$ As discussed above, examples of both of these scenarios are present within the phases predicted here.

In our discussion it is also instructive to consider the evolution of the low temperature phases of HF under pressure. At ambient conditions the $C m c 2_{1}$ symmetry HF phase contains planar zigzag chains of hydrogen-bonded molecules that are held together via van der Waals forces. ${ }^{93}$ Between 25-143 GPa a $\mathrm{Cmcm}$ symmetry structure where each fluorine atom is symmetrically bonded to two hydrogen atoms is preferred. ${ }^{53,94}$ First principles calculations have predicted a further transition to a Pnma symmetry structure with nearly symmetric $\mathrm{H}-\mathrm{F}^{53}$ bonds that is stable until at least $900 \mathrm{GPa}{ }^{95}$

In order to better understand the bonding within the $(\mathrm{F}-\mathrm{H}-$ $\mathrm{F})^{-}$and $(\mathrm{FH}-\mathrm{F}-\mathrm{HF})^{-}$anions we calculated the negative of the crystal orbital Hamilton populations between select atoms integrated to the Fermi level (-iCOHP) because they can be used to gauge the bond strength. The results obtained for the Li-F-H phases can be compared with values calculated for a symmetric $(\mathrm{F}-\mathrm{H}-\mathrm{F})^{-}$molecule optimized in the gas phase at $0 \mathrm{GPa}$, and Pnma HF optimized at $300 \mathrm{GPa}$ (Table I).

$\mathrm{LiF}_{2} \mathrm{H}, \mathrm{Li}_{3} \mathrm{~F}_{4} \mathrm{H}$ and $\mathrm{Li}_{2} \mathrm{~F}_{3} \mathrm{H}$ contain linear bifluoride anions with equivalent $\mathrm{F}-\mathrm{H}$ bond lengths, and therefore they can be considered $3 c-4 e$ bonded species. Because the neighboring $\mathrm{Li}^{+}$cations are arranged symmetrically about the $(\mathrm{F}-$ $\mathrm{H}-\mathrm{F})^{-}$anions, the geometry of these molecular motifs are not distorted via electrostatic interactions. The $\mathrm{F}-\mathrm{H}$ bond lengths in these species are shorter than in the bifluoride anion in the gas phase, and the bond strengths, as measured

TABLE I. Distances between fluorine and hydrogen atoms, and F$\mathrm{H}-\mathrm{F}$ angles within the bifluoride anion in the gas phase, as well as Pnma HF at $300 \mathrm{GPa}$, and their corresponding crystal orbital Hamilton populations integrated to the Fermi level (-iCOHP). These same quantities are given for the bifluoride ions present within the $\mathrm{Li}-\mathrm{F}-\mathrm{H}$ phases at $300 \mathrm{GPa}$.

\begin{tabular}{cccccc}
\hline \hline System & \multicolumn{5}{c}{ Distance $(\AA)$} \\
& F1-H & F2-H & F-H-F & F1-H & F2-H \\
\hline$(\mathrm{F}-\mathrm{H}-\mathrm{F})^{-}$ & 1.160 & 1.160 & 180.0 & 3.61 & 3.61 \\
$\mathrm{HF}$ & 1.047 & 1.048 & 175.3 & 7.46 & 7.09 \\
$\mathrm{LiF}_{2} \mathrm{H}^{a}$ & 1.037 & 1.037 & 180.0 & 6.89 & 6.89 \\
$\mathrm{LiF}_{2} \mathrm{H}^{a}$ & 1.034 & 1.067 & 165.3 & 7.10 & 6.33 \\
$\mathrm{Li}_{3} \mathrm{~F}_{4} \mathrm{H}$ & 1.041 & 1.041 & 180.0 & 6.95 & 6.95 \\
$\mathrm{LiF}_{4} \mathrm{H}_{4}$ & 1.035 & 1.042 & 175.5 & 7.33 & 7.06 \\
$\mathrm{Li}_{2} \mathrm{~F}_{3} \mathrm{H}$ & 1.041 & 1.041 & 180.0 & 6.96 & 6.96 \\
$\mathrm{LiF}_{3} \mathrm{H}$ & 1.014 & 1.079 & 180.0 & 7.81 & 6.22 \\
\hline \hline
\end{tabular}

${ }^{a}$ Linear and bent bifluoride anions are present in this phase. 
(a)

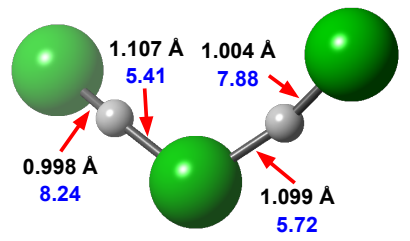

(b)

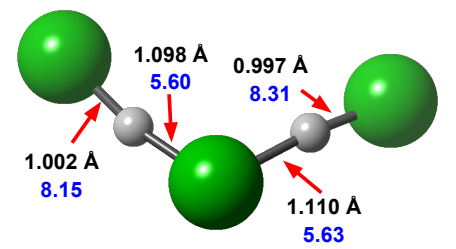

FIG. 2. The molecular conformation of the (FH-F-HF) ${ }^{-}$anions taken from (a) $\mathrm{LiF}_{3} \mathrm{H}_{2}$ and (b) $\mathrm{LiF}_{4} \mathrm{H}_{4}$ at $300 \mathrm{GPa}$. The values given in black are the bond lengths in $\AA$, and the values colored in blue are the $-\mathrm{iCOHPs}$ in $\mathrm{eV} /$ bond.

by the -iCOHPs, are therefore almost twice as large, in-line with the bond strengthening that is expected to occur because of increased orbital overlap under pressure. ${ }^{96}$ The calculated $\mathrm{F}-\mathrm{H}$ bond strengths and lengths in the Li-F-H ternaries are not too different from those found in solid HF at the same pressure. In addition to the $D_{\infty h}$ symmetry $(\mathrm{F}-\mathrm{H}-\mathrm{F})^{-}$unit, $\mathrm{LiF}_{2} \mathrm{H}$ also contains a bifluoride anion that is bent and asymmetric because of electrostatic interactions with neighboring $\mathrm{Li}^{+}$cations and other $(\mathrm{F}-\mathrm{H}-\mathrm{F})^{-}$species. Both sets of $\mathrm{H}-\mathrm{F}$ bonds are stronger and shorter than in the gas phase molecule. Asymmetric (F-H-F) ${ }^{-}$also comprise $\mathrm{LiF}_{4} \mathrm{H}_{4}$, whose bifluoride anion is slightly bent, and $\mathrm{LiF}_{3} \mathrm{H}$, where it remains linear. The bond strengths in the ternaries typically increase with decreasing bond lengths.

Although the asymmetric H-F bonds within the 1D zigzag chains in HF do not have a significantly different bond length, their different chemical environments affect their strengths with calculated -iCOHPs of 7.46 and $7.09 \mathrm{eV} /$ bond. Generally speaking the H-F bond distances in Pnma HF are slightly larger than in the ternaries, but the -iCOHPs are also larger. This suggests that the complicated chemical environment in the ternary phases with the presence of Lewis acid species destabilizes the bifluoride anions.

Figure 2 illustrates the H-F bond lengths and associated $-\mathrm{iCOHPs}$ in the more complicated $(\mathrm{FH}-\mathrm{F}-\mathrm{HF})^{-}$units that are present in $\mathrm{LiF}_{3} \mathrm{H}_{2}$ and $\mathrm{LiF}_{4} \mathrm{H}_{4}$. In both cases the $\mathrm{H}-\mathrm{F}_{\text {terminal }}$ bonds are shorter and stronger than in $\mathrm{HF}$ or in any of the ternaries, whereas the $\mathrm{H}-\mathrm{F}_{\text {middle }}$ are longer and weaker $(\sim 1 \AA$ and 7.9-8.3 eV/bond vs. $\sim 1.1 \AA$ and 5.4-5.7 eV/bond). This coincides with the 1 atm picture where the $(\mathrm{FH}-\mathrm{F}-\mathrm{HF})^{-}$motifs are viewed as being composed of two HF units hydrogen bonded to a central $\mathrm{F}^{-} .81$

Bader charges were calculated to verify the formal charges assigned. The results, provided in Table S3, show that in all of the Li-F-H phases the Bader charge on Li fell between +0.83 and +0.86 , in-line with the +1 oxidation state. The charges on the fluorine and hydrogen atoms within the $\mathrm{H}_{n} \mathrm{~F}_{n+1}^{-}$anions typically ranged from -0.74 to -0.80 , and +0.73 to +0.76 , respectively, which is in-line with the values computed for $\mathrm{HF}$ at the same pressure, $-/+0.75$. Generally speaking, the overall charges on the $\mathrm{H}_{n} \mathrm{~F}_{n+1}^{-}$anions fell between -0.76 to -0.85 , and the charges on the fluorine atoms comprising the LiF slabs were somewhat more negative than in the molecular motifs, $0.82 / 0.83$. Only one phase, $\mathrm{LiF}_{3} \mathrm{H}$, deviated from these trends. The reason for this turns out to be key for the metallicity of this phase, as described below.

\section{Electronic Structure and Properties}

Figure 3 plots the electron localization functions (ELFs) of $\mathrm{LiF}_{3} \mathrm{H}_{2}, \mathrm{LiF}_{2} \mathrm{H}$, and $\mathrm{LiF}_{4} \mathrm{H}_{4}$ because they are representative of all of the structural motifs observed. $\mathrm{LiF}_{3} \mathrm{H}_{2}$ and $\mathrm{LiF}_{4} \mathrm{H}_{4}$ contain the $(\mathrm{FH}-\mathrm{F}-\mathrm{HF})^{-}$anion; $\mathrm{LiF}_{4} \mathrm{H}_{4}$ also accommodates the $\mathrm{H}_{3}^{+}$unit and asymmetric $(\mathrm{F}-\mathrm{H}-\mathrm{F})^{-}$; and $\mathrm{LiF}_{2} \mathrm{H}$ contains both symmetric and asymmetric $(\mathrm{F}-\mathrm{H}-\mathrm{F})^{-}$. Because the planes of the contours chosen do not necessarily coincide with planes passing through the bifluoride anions and $\mathrm{H}_{3}^{+}$, and because a single plane cannot pass through (FH-F$\mathrm{HF})^{-}$, some discontinuities are present in the ELF contour maps. Therefore, isosurface plots are also provided in Figures S6-8. Nonetheless, the ELF plots clearly show high regions of localization around the fluorine atoms, with slightly more density localized along the F-H contacts that are shorter as compared to those that are longer. Moreover, the ELF is high around the $\mathrm{H}_{3}^{+}$molecule because of its $3 \mathrm{c}-2 \mathrm{e}$ bond.

The electronic band structure and densities of states (DOS) plots of $\mathrm{LiF}_{3} \mathrm{H}_{2}, \mathrm{LiF}_{2} \mathrm{H}, \mathrm{Li}_{3} \mathrm{~F}_{4} \mathrm{H}, \mathrm{LiF}_{4} \mathrm{H}_{4}, \mathrm{Li}_{2} \mathrm{~F}_{3} \mathrm{H}$, and $\mathrm{LiF}_{3} \mathrm{H}$ at $300 \mathrm{GPa}$ are provided in Figure 4. As expected, the F $p$ orbitals contribute the most to the occupied DOS. The bottom of the conduction band, on the other hand, contains almost equal contributions from $\mathrm{F} 2 p, \mathrm{H} 1 s$, and Li $2 s$ states, which is consistent with the Bader charges indicating that both $\mathrm{H}$ and $\mathrm{Li}$ transfer electrons to the most electronegative element present. Except for $\mathrm{LiF}_{3} \mathrm{H}$, all of the Li-F-H phases considered here have large band gaps, which are listed in the DOS plots, ranging from 10.4 to $14.4 \mathrm{eV}$ within the PBE functional. The band gap for the $B 1 \mathrm{LiF}$ phase calculated at this level of theory is $15.9 \mathrm{eV}$ at $300 \mathrm{GPa}$, suggesting that the presence of hydrogen in the ternary phases reduces the band gap slightly. The large band gaps of these ternary hydrides suggest that they remain transparent at $300 \mathrm{GPa}$.

Only one phase, $P 2_{1} / m \mathrm{LiF}_{3} \mathrm{H}$, was calculated to be metallic at $300 \mathrm{GPa}$. The DOS at the Fermi level, $E_{F}$, is almost completely due to the F $2 p$ orbitals. The metallicity of this phase is not a result of pressure-induced band broadening, but rather a result of the dissociation of $\mathrm{F}_{2}$ molecules and the electron count in the compound. Between 70-2500 GPa diatomic fluorine is predicted to adopt the $\mathrm{Cmca}$ structure, followed by a transformation to a novel metallic phase with $\mathrm{P}_{2} / \mathrm{mmc}_{\mathrm{m}}$ symmetry. ${ }^{49}$ In contrast, experiments have shown that iodine becomes monoatomic, metallic and superconduct- 

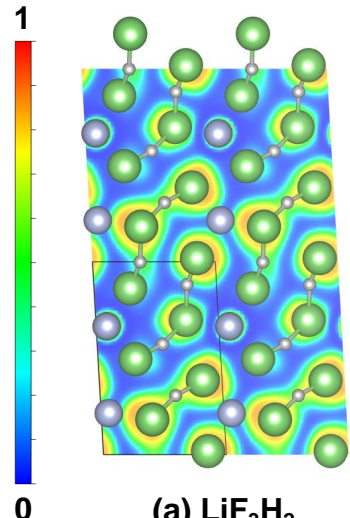

(a) $\mathrm{LiF}_{3} \mathrm{H}_{2}$

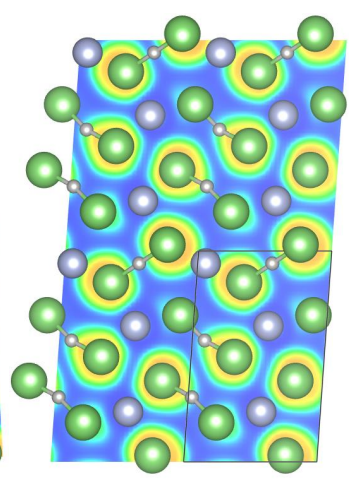

(b) $\mathrm{LiF}_{2} \mathrm{H}$

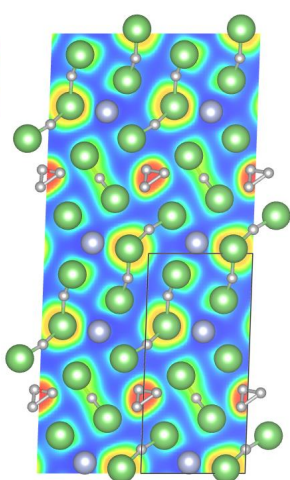

(c) $\mathrm{LiF}_{4} \mathrm{H}_{4}$

FIG. 3. Contour maps of the electron localization function (ELF) at $300 \mathrm{GPa}$ shown for $2 \times 2 \times 2$ supercells of (a) $P \overline{1} \mathrm{LiF}_{3} \mathrm{H}_{2}(100)$ plane, (b) $P \overline{1} \mathrm{LiF}_{2} \mathrm{H}$ (001) plane, and (c) $P 1 \mathrm{LiF}_{4} \mathrm{H}_{4}$ (100) plane. $\mathrm{Li} / \mathrm{F} / \mathrm{H}$ atoms are colored blue/green/white.

ing with a $T_{c}$ of $1.2 \mathrm{~K}$ already at $28 \mathrm{GPa} \cdot{ }^{97-99} \mathrm{In}$ the full ionic picture the formula of $P 2_{1} / m \mathrm{LiF}_{3} \mathrm{H}$ can be written as $\mathrm{Li}^{+}\left(\mathrm{FHF}^{-}\right) \mathrm{F}^{0}$. This phase could be insulating if fluorine were present as a diatomic molecule. However, the Bader charges yield $\mathrm{Li}^{+0.85}\left(\mathrm{FHF}^{-0.52}\right) \mathrm{F}^{-0.33}$, and the nearest neighbor distance between two fluorine atoms in the $\mathrm{LiF}$ slab separating the bifluoride layers measures $1.610 \AA$, as compared to the F$\mathrm{F}$ bond length of $1.393 \AA$ in $\mathrm{Cmca} \mathrm{F}_{2}$ at the same pressure. Since $\mathrm{F}_{2}$ molecules are not found in $P 2_{1} / m \mathrm{LiF}_{3} \mathrm{H}$, an extra electron per formula unit would be required to render it a wide-gap insulator.

Because $P 2_{1} / m \mathrm{LiF}_{3} \mathrm{H}$ is metallic, we calculated its electron-phonon coupling (EPC) parameter, $\lambda$, and estimated its $T_{c}$ using the Allen-Dynes modified McMillan equation ${ }^{100}$, $T_{c}=\frac{\omega_{\log }}{1.2} \exp \left[-\frac{1.04(1+\lambda)}{\lambda-\mu^{*}(1+0.62 \lambda)}\right]$, where $\omega_{\log }$ is the logarithmic average frequency, and $\mu^{*}$ is the Coulomb pseudopotential, often assumed to be between $\sim 0.1-0.13$. The EPC parameter was calculated to be 0.27 and $\omega_{\log }$ was $695.2 \mathrm{~K}$ yielding an estimated $T_{c}$ of $0.092-0.007 \mathrm{~K}$. Thus, in contrast with Gilman's prediction ${ }^{46}$ none of the low-lying metastable Li-F$\mathrm{H}$ ternary phases at $300 \mathrm{GPa}$ are good candidates for pressureinduced hydride based superconductors. Finally, to aid future characterization of these phases, should they ever be synthesized, we present calculated infrared spectra for $\mathrm{HF}, \mathrm{LiF}_{3} \mathrm{H}_{2}$, $\mathrm{LiF}_{2} \mathrm{H}, \mathrm{Li}_{3} \mathrm{~F}_{4} \mathrm{H}, \mathrm{LiF}_{4} \mathrm{H}_{4}, \mathrm{Li}_{2} \mathrm{~F}_{3} \mathrm{H}$, and $\mathrm{LiF}_{3} \mathrm{H}$ in Figures. S2935 .

\section{CONCLUSION}

Using evolutionary crystal structure searches we predict a number of metastable phases, $\mathrm{LiF}_{3} \mathrm{H}_{2}, \mathrm{LiF}_{2} \mathrm{H}, \mathrm{Li}_{3} \mathrm{~F}_{4} \mathrm{H}$, $\mathrm{LiF}_{4} \mathrm{H}_{4}, \mathrm{Li}_{2} \mathrm{~F}_{3} \mathrm{H}$ and $\mathrm{LiF}_{3} \mathrm{H}$ that are within $50 \mathrm{meV} /$ atom of the ternary convex hull within the static-lattice approximation. These potentially synthesizable phases contain the bifluoride anion, (F-H-F) ${ }^{-}$, or the longer (FH-F-HF) ${ }^{-}$molecular motif, along with $\mathrm{Li}^{+}$cations. $\mathrm{LiF}_{4} \mathrm{H}_{4}$ additionally features an
$\mathrm{H}_{3}^{+}$counter-cation, and $\mathrm{LiF}$ slabs are present within $\mathrm{Li}_{3} \mathrm{~F}_{4} \mathrm{H}$. The bonding within the $\mathrm{H}_{n} \mathrm{~F}_{n+1}^{-}$motifs encountered here is analyzed, and it ranges from multi-centered interactions to those containing classic two-centered two-electron bonds and a $\mathrm{H} \cdots \mathrm{F}^{-}$hydrogen bonds. With the exception of $\mathrm{LiF}_{3} \mathrm{H}$, all of the low-lying predicted compounds are computed to be wide gap insulators. Our calculations suggest that other analogous phases with $n>2$ could potentially be metastable at these conditions. For example, exploratory calculations on the $\mathrm{LiF}_{4} \mathrm{H}_{3}$ stoichiometry predict a structure that contains bent and asymmetric bifluoride anions, as well as infinite HF chains with nearly equal bond lengths, whose enthalpy lies $21.7 \mathrm{meV} /$ atom above the convex hull. This studies provides the basis for future work exploring the finite temperature stability of Li-F-H phases under high pressure, with the inclusion of anharmonic effects, which are known to be important for light element systems at these conditions.

\section{ACKNOWLEDGEMENT}

E.Z. and T.B. acknowledge the NSF (DMR-1827815), and R.H. acknowledges the NSF (DMR-1933622) for financial support. We thank the U.S. Department of Energy, National Nuclear Security Administration, through the ChicagoDOE Alliance Center under Cooperative Agreement DENA0003975. Calculations were performed at the Center for Computational Research at SUNY Buffalo. ${ }^{101}$ We are grateful to Sebastien Hamel for useful discussions. A.S. notes LLNLJRNL-817757.

\section{DATA AVAILABILITY}

The data of this study are available from the corresponding author upon request. 
(a)

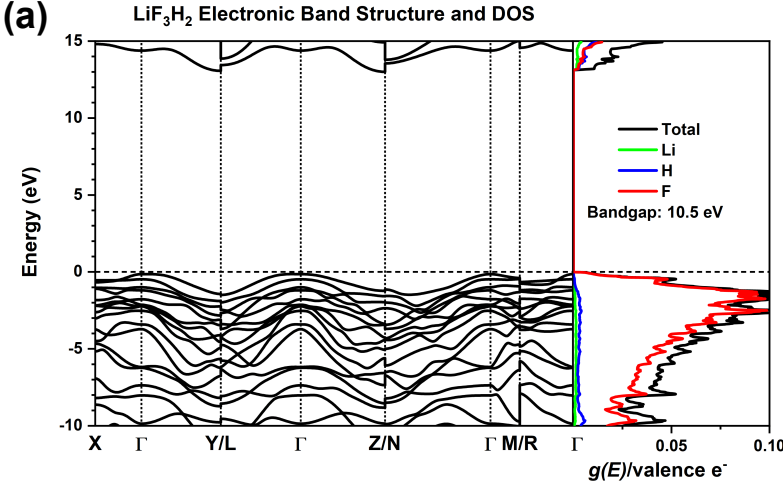

(c)

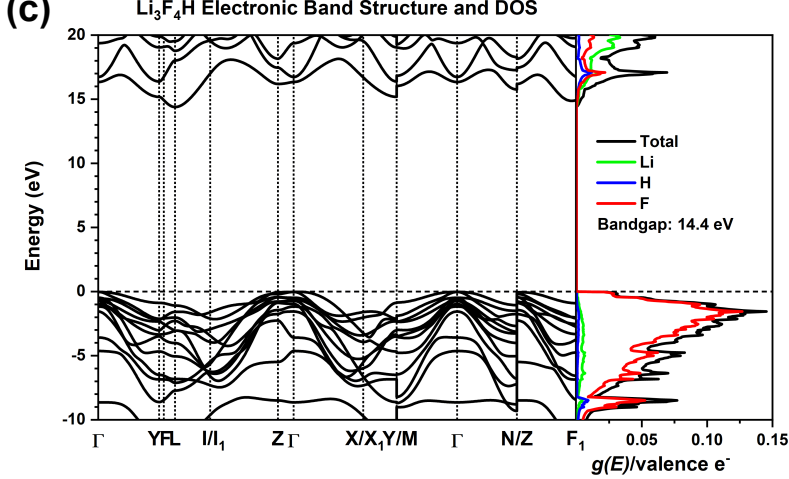

(e)

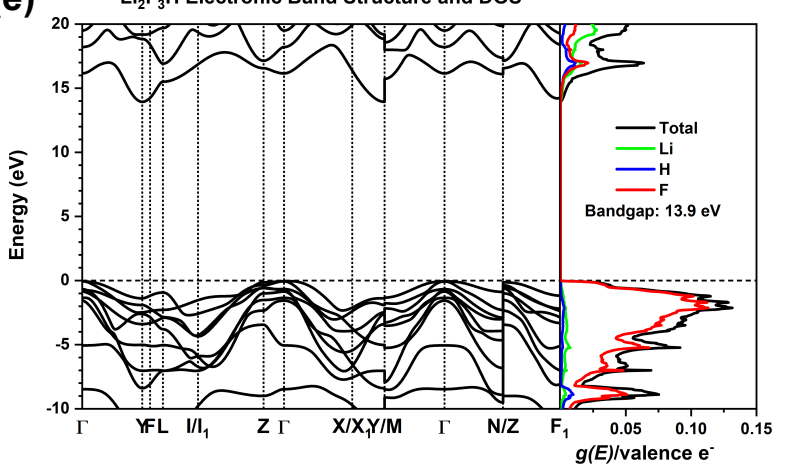

(b)

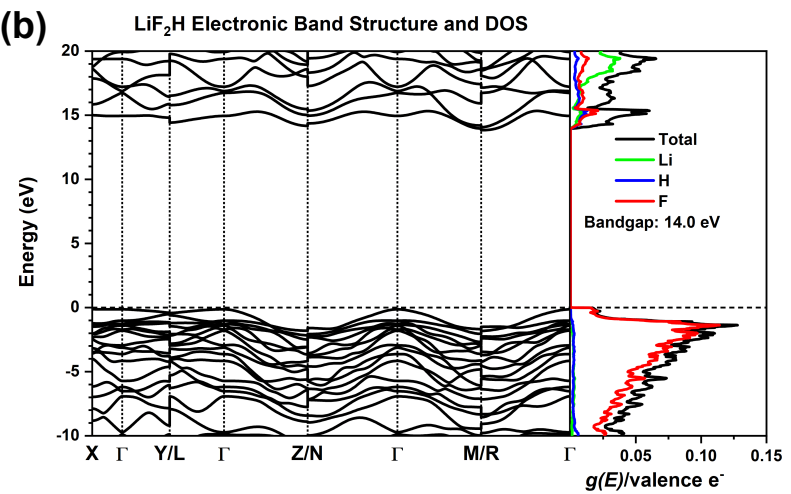

(d)

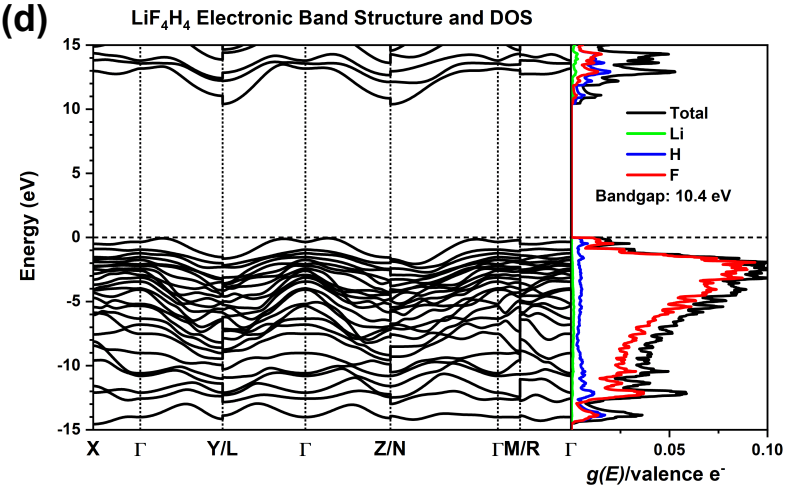

(f)

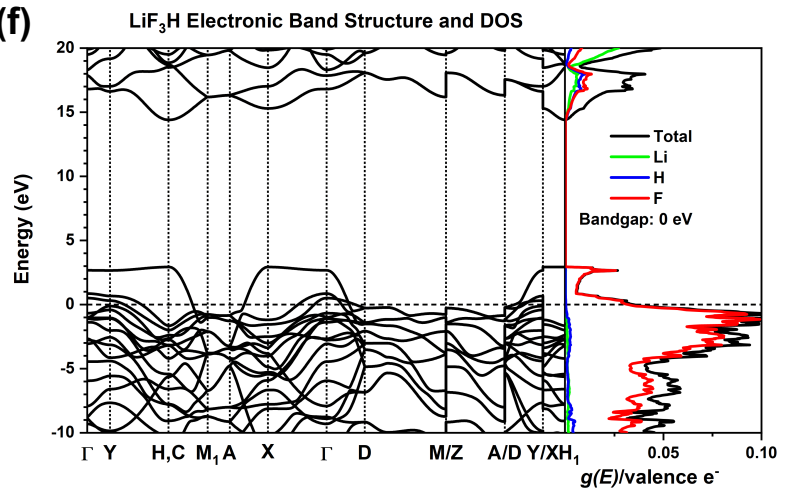

FIG. 4. Electronic band structure and projected densities of states (PDOS) of (a) $P \overline{1} \mathrm{LiF}_{3} \mathrm{H}_{2}$, (b) $P \overline{1} \mathrm{LiF}_{2} \mathrm{H}$, (c) $C 2 / m \mathrm{Li}_{3} \mathrm{~F}_{4} \mathrm{H}$, (d) $P 1$ $\mathrm{LiF}_{4} \mathrm{H}_{4}$, (e) $C 2 / m \mathrm{Li}_{2} \mathrm{~F}_{3} \mathrm{H}$, and (f) $P 2_{1} / m \mathrm{LiF}_{3} \mathrm{H}$ at $300 \mathrm{GPa}$. In (a-e) the top of the valence band is set to $0 \mathrm{eV}$, whereas in (f) the Fermi level is set to $0 \mathrm{eV}$.

\section{REFERENCES}

${ }^{1}$ A. Banos, N. J. Harker, and T. B. Scott, "A review of uranium corrosion by hydrogen and the formation of uranium hydride," Corros. Sci. 136, 129-147 (2018).

${ }^{2}$ M. B. Ley, L. H. Jepsen, Y.-S. Lee, Y. W. Cho, J. M. Bellosta von Colbe, M. Dornheim, M. Rokni, J. O. Jensen, M. Sloth, Y. Filinchuk, E. ErikJørgensen, F. Besenbacher, and T. R. Jensen, "Complex hydrides for hydrogen storagenew perspectives," Mater. Today 17, 122-128 (2014).

${ }^{3}$ S. Kim, H. Oguchi, N. Toyama, T. Sato, S. Takagi, T. Otomo, D. Arunkumar, N. Kuwata, J. Kawamura, and S. Orimo, "A complex hydride lithium superionic conductor for high-energy-density all-solid-state lithium metal batteries," Nat. Commun. 10, 1081 (2019).

${ }^{4}$ Y. Song, "New perspectives on potential hydrogen storage materials using high pressure," Phys. Chem. Chem. Phys. 15, 14524-14547 (2013).

${ }^{5}$ V. V. Struzhkin, B. Militzer, W. L. Mao, H. Mao, and R. J. Hemley, "Hydrogen storage in molecular clathrates," Chem. Rev. 107, 4133-4151 (2007).

${ }^{6} \mathrm{~T}$. Bi, N. Zarifi, T. Terpstra, and E. Zurek, "The search for superconductivity in high pressure hydrides," in Elsevier Reference Module in Chemistry, Molecular Sciences and Chemical Engineering, edited by J. Reedijk (Elsevier, Waltham, MA, 2019) pp. 1-36.

${ }^{7}$ E. Zurek and T. Bi, "High-temperature superconductivity in alkaline and rare earth polyhydrides at high pressure: 
A theoretical perspective," J. Chem. Phys. 150, 050901 (2019).

${ }^{8}$ E. Zurek, "Hydrides of the alkali metals and alkaline earth metals under pressure," Comments Inorg. Chem. 37, 78-98 (2017).

${ }^{9}$ A. Shamp and E. Zurek, "Superconductivity in hydrides doped with main group elements under pressure," Nov. Supercond. Mater. 3, 14-22 (2017).

${ }^{10}$ D. Duan, Y. Liu, F. Tian, D. Li, X. Huang, Z. Zhao, H. Yu, B. Liu, W. Tian, and T. Cui, "Pressure-induced metallization of dense $\left(\mathrm{H}_{2} \mathrm{~S}\right)_{2} \mathrm{H}_{2}$ with high- $T_{c}$ superconductivity," Sci. Rep. 4, 6968 (2014).

${ }^{11}$ H. Liu, I. I. Naumov, R. Hoffmann, N. W. Ashcroft, and R. J. Hemley, "Potential high- $T_{c}$ superconducting lanthanum and yttrium hydrides at high pressure," Proc. Natl. Acad. Sci. U.S.A. 114, 6990-6995 (2017).

${ }^{12}$ F. Peng, Y. Sun, C. J. Pickard, R. J. Needs, Q. Wu, and Y. Ma, "Hydrogen clathrate structures in rare earth hydrides at high pressures: possible route to room-temperature superconductivity," Phys. Rev. Lett 119, 107001 (2017).

${ }^{13}$ A. P. Drozdov, M. I. Eremets, I. A. Troyan, V. Ksenofontov, and S. I. Shylin, "Conventional superconductivity at 203 kelvin at high pressures in the sulfur hydride system," Nature 525, 73-76 (2015).

${ }^{14}$ Z. M. Geballe, H. Liu, A. K. Mishra, M. Ahart, M. Somayazulu, Y. Meng, M. Baldini, and R. J. Hemley, "Synthesis and stability of lanthanum superhydrides," Angew. Chem. Int. Ed. 57, 688-692 (2017).

${ }^{15}$ M. Somayazulu, M. Ahart, A. K. Mishra, Z. M. Geballe, M. Baldini, Y. Meng, V. V. Struzhkin, and R. J. Hemley, "Evidence for superconductivity above $260 \mathrm{~K}$ in lanthanum superhydride at megabar pressures," Phys. Rev. Lett. 122, 027001 (2019).

${ }^{16}$ A. P. Drozdov, P. P. Kong, V. S. Minkov, S. P. Besedin, M. A. Kuzovnikov, S. Mozaffari, L. Balicas, F. F. Balakirev, D. E. Graf, V. B. Prakapenka, E. Greenberg, D. A. Knyazev, M. Tkacz, and M. I. Eremets, "Superconductivity at $250 \mathrm{~K}$ in lanthanum hydride under high pressures," Nature 569, 528-531 (2019).

${ }^{17}$ Z. Shao, D. Duan, Y. Ma, H. Yu, H. Song, H. Xie, D. Li, F. Tian, B. Liu, and T. Cui, "Ternary superconducting cophosphorus hydrides stabilized via lithium," npj. Comput. Mater. 5, 1-8 (2019).

${ }^{18}$ X. Li, Y. Xie, Y. Sun, P. Huang, H. Liu, C. Chen, and Y. Ma, "Chemically tuning stability and superconductivity of $\mathrm{P}-\mathrm{H}$ compounds," J. Phys. Chem. Lett. 11, 935-939 (2020).

${ }^{19}$ Y. Sun, J. Lv, Y. Xie, H. Liu, and Y. Ma, "Route to a superconducting phase above room temperature in electrondoped hydride compounds under high pressure," Phys. Rev. Lett. 123, 097001 (2019).

${ }^{20}$ W. Cui, T. Bi, J. Shi, Y. Li, H. Liu, E. Zurek, and R. J. Hemley, "Route to high- $T_{c}$ superconductivity via $\mathrm{CH}_{4}$ intercalated $\mathrm{H}_{3} \mathrm{~S}$ hydride perovskites," Phys. Rev. B 101, 134504 (2020).

${ }^{21}$ Y. Sun, Y. Tian, B. Jiang, X. Li, H. Li, T. Iitaka, X. Zhong, and Y. Xie, "Computational discovery of a dynamically stable cubic $\mathrm{SH}_{3}$-like high-temperature superconductor at $100 \mathrm{GPa}$ via $\mathrm{CH}_{4}$ intercalation,” Phys. Rev. B 101, 174102
(2020).

${ }^{22}$ B. Liu, W. Cui, J. Shi, L. Zhu, J. Chen, S. Lin, R. Su, J. Ma, K. Yang, M. Xu, J. Hao, A. P. Durajski, J. Qi, Y. Li, and Y. Li, "Effect of covalent bonding on the superconducting critical temperature of the H-S-Se system," Phys. Rev. B 98, 174101 (2018).

${ }^{23}$ F. Tian, D. Li, D. Duan, X. Sha, Y. Liu, T. Yang, B. Liu, and T. Cui, "Predicted structures and superconductivity of hypothetical $\mathrm{Mg}-\mathrm{CH}_{4}$ compounds under high pressures," Mater. Res. Express 2, 046001 (2015).

${ }^{24}$ F. Fan, D. A. Papaconstantopoulos, M. J. Mehl, and B. M. Klein, "High-temperature superconductivity at high pressures for $\mathrm{H}_{3} \mathrm{Si}_{x} \mathrm{P}_{1-x}, \mathrm{H}_{3} \mathrm{P}_{x} \mathrm{~S}_{1-x}$, and $\mathrm{H}_{3} \mathrm{Cl}_{x} \mathrm{~S}_{1-x}$, J. Phys. Chem. Solids 99, 105-110 (2016).

${ }^{25}$ Y. Yan, T. Bi, N. Geng, X. Wang, and E. Zurek, "A metastable $\mathrm{CaSH}_{3}$ phase composed of HS honeycomb sheets that is superconducting under pressure," J. Phys. Chem. Lett. 11, 9629-9636 (2020).

${ }^{26}$ X. Liang, A. Bergara, L. Wang, B. Wen, Z. Zhao, X.-F. Zhou, J. He, G. Gao, and Y. Tian, "Potential high- $T_{c}$ superconductivity in $\mathrm{CaYH}_{12}$ under pressure," Phys. Rev. B 99, 100505(R) (2019).

${ }^{27}$ E. Snider, N. Dasenbrock-Gammon, R. McBride, M. Debessai, H. Vindana, K. Vencatasamy, K. V. Lawler, A. Salamat, and R. P. Dias, "Room-temperature superconductivity in a carbonaceous sulfur hydride," Nature 586, 373-377 (2020).

${ }^{28}$ L. E. Kirsch, S. J. Ali, D. E. Fratanduono, R. G. Kraus, D. G. Braun, A. Fernandez-Panella, R. F. Smith, J. M. McNaney, and J. H. Eggert, "Refractive index of lithium fluoride to 900 gigapascal and implications for dynamic equation of state measurements," J. Appl. Phys. 125, 175901 (2019).

${ }^{29}$ T. Ao, M. D. Knudson, J. R. Asay, and J. P. Davis, "Strength of lithium fluoride under shockless compression to 114 GPa," J. Appl. Phys. 106, 103507 (2009).

${ }^{30}$ J. Liu, L. Dubrovinsky, T. Boffa Ballaran, and W. Crichton, "Equation of state and thermal expansivity of $\mathrm{LiF}$ and NaF," High Pressure Res. 27, 483-489 (2007).

${ }^{31}$ H. Dong, S. M. Dorfman, C. M. Holl, Y. Meng, V. B. Prakapenka, D. He, and T. S. Duffy, "Compression of lithium fluoride to $92 \mathrm{GPa}$," High Pressure Res. 34, 39-48 (2014).

${ }^{32}$ D. E. Fratanduono, T. R. Boehly, M. A. Barrios, D. D. Meyerhofer, J. H. Eggert, R. F. Smith, D. G. Hicks, P. M. Celliers, D. G. Braun, and G. W. Collins, "Refractive index of lithium fluoride ramp compressed to $800 \mathrm{GPa}$," J. Appl. Phys. 109, 123521 (2011).

${ }^{33}$ L. M. Barker and R. E. Hollenbach, "Laser interferometer for measuring high velocities of any reflecting surface," J. Appl. Phys 43, 4669 (1972).

${ }^{34}$ Q. Liu, X. Zhou, X. Zeng, and S. N. Luo, "Sound velocity, equation of state, temperature and melting of LiF single crystals under shock compression,' J. Appl. Phys. 117, 045901 (2015).

${ }^{35}$ J. P. Davis, M. D. Knudson, L. Schulenburger, and S. D. Crockett, "Mechanical and optical response of [100] lithium fluoride to multi-megabar dynamic pressures," J. 
Appl. Phys. 120, 165901 (2016).

${ }^{36}$ P. C. Myint, E. L. Shi, S. Hamel, H. Cynn, Z. Jenei, M. J. Lipp, W. J. Evans, and M. C. Akin, "Two-phase equation of state for lithium fluoride," J. Chem. Phys. 150, 074506 (2019).

${ }^{37}$ J. L. Wise and L. C. Chhabildas, "Laser interferometer measurements of refractive index in shock-compressed materials," in Shock Waves in Condensed Matter (Springer, 1986) pp. 441-454.

${ }^{38}$ K. P. Driver and B. Militzer, "First-principles simulations of warm dense lithium fluoride," Phys. Rev. E 95, 043205 (2017).

${ }^{39}$ C. D. Spataru, L. Shulenburger, and L. X. Benedict, “ $A b$ initio many-body Green's function calculations of optical properties of LiF at high pressures," Phys. Rev. B 92, 245117 (2015).

${ }^{40}$ N. A. Smirnov, "Ab initio calculations of the thermodynamic properties of LiF crystal," Phys. Rev. B 83, 014109 (2011).

${ }^{41}$ Z.-H. Sun, J. Dong, and Y.-W. Xia, "First-principles calculations of the structural, electronic, and optical properties of LiF up to $300 \mathrm{GPa}$," Physica B 406, 3660-3665 (2011).

${ }^{42}$ X. Duan, C. Zhang, Z. Guan, L. Sun, X. Peng, H. Liu, W. Yang, Y. Li, H. Zhang, Q. Ye, J. Yang, S. Jiang, and Z. Wang, "Transparency measurement of lithium fluoride under laser-driven accelerating shock loading," J. Appl. Phys 128, 015902 (2020).

${ }^{43}$ L. He, M. J. Tang, J. Yin, X. M. Zhou, W. J. Zhu, F. S. Liu, and D. W. He, "Effects of the vacancy point-defect on the refractive index and equation of state (EOS) of $\mathrm{LiF}$ at high pressure: A first principles investigation," Physica B Condens. Matter 407, 694-697 (2012).

${ }^{44}$ M. D. Knudson, M. P. Desjarlais, A. Becker, R. W. Lemke, K. R. Cochrane, M. E. Savage, D. E. Bliss, T. R. Mattsson, and R. Redmer, "Direct observation of an abrupt insulatorto-metal transition in dense liquid deuterium," Science $\mathbf{3 4 8}$, 1455-1460 (2015).

${ }^{45}$ P. M. Celliers, M. Millot, S. Brygoo, R. Stewart McWilliams, D. E. Fratanduono, R. J. Rygg, A. F. Goncharov, P. Loubeyre, J. H. Eggert, J. L. Peterson, N. B. Meezan, S. Le Pape, G. W. Collins, R. Jeanloz, and R. J. Hemley, "Insulator-metal transition in dense fluid deuterium," Science 361, 677-682 (2018).

${ }^{46}$ J. J. Gilman, "Lithium dihydrogen fluoride - an approach to metallic hydrogen," Phys. Rev. Lett. 26, 546 (1971).

${ }^{47}$ J. Lv, Y. Wang, L. Zhu, and Y. Ma, "Predicted novel highpressure phases of lithium," Phys. Rev. Lett. 106, 015503 (2011).

${ }^{48}$ C. J. Pickard and R. J. Needs, "Structure of phase III of solid hydrogen," Nat. Phys. 3, 473-476 (2007).

${ }^{49}$ M. A. Olson, S. Bhatia, P. Larson, and B. Militzer, "Prediction of chlorine and fluorine crystal structures at high pressure using symmetry driven structure search with geometric constraints,' J. Chem. Phys. 153, 094111 (2020).

${ }^{50}$ M. J. van Setten, V. A. Popa, G. A. de Wijs, and G. Brocks, "Electronic structure and optical properties of lightweight metal hydrides," Phys. Rev. B 75, 035204 (2007).
${ }^{51}$ E. Zurek, R. Hoffmann, N. W. Ashcroft, A. R. Oganov, and A. O. Lyakhov, "A little bit of lithium does a lot for hydrogen,” Proc. Natl. Acad. Sci. U.S.A. 106, 17640-17643 (2009).

${ }^{52}$ D. Duan, X. Huang, F. Tian, Y. Liu, D. Li, H. Yu, B. Liu, W. Tian, and T. Cui, "Predicted formation of $\mathrm{H}_{3}^{+}$in solid halogen polyhydrides at high pressures," J. Phys. Chem. A 119, 11059-11065 (2015).

${ }^{53}$ L. Zhang, Y. Wang, X. Zhang, and Y. Ma, "High-pressure phase transitions of solid $\mathrm{HF}, \mathrm{HCl}$, and $\mathrm{HBr}$ : An ab initio evolutionary study," Phys. Rev. B 82, 014108 (2010).

${ }^{54}$ D. C. Lonie and E. Zurek, "XTALOPT: An open-source evolutionary algorithm for crystal structure prediction," Comput. Phys. Commun. 182, 372-387 (2011).

${ }^{55}$ Z. Falls, D. C. Lonie, P. Avery, A. Shamp, and E. Zurek, "XTALOPT version r9: An open-source evolutionary algorithm for crystal structure prediction," Comput. Phys. Commun. 199, 178-179 (2016).

${ }^{56}$ Z. Falls, P. Avery, X. Wang, K. P. Hilleke, and E. Zurek, "The XTALOPT evolutionary algorithm for crystal structure prediction," J. Phys. Chem. C (2020), 10.1021/acs.jpcc.0c09531.

${ }^{57}$ D. C. Lonie and E. Zurek, "Identifying duplicate crystal structures: XTALCOMP, an open-source solution," Comput. Phys. Commun. 183, 690-697 (2012).

${ }^{58}$ G. Kresse and J. Hafner, "Ab initio molecular dynamics for liquid metals,” Phys. Rev. B 47, 558(R) (1993).

${ }^{59}$ J. P. Perdew, K. Burke, and M. Ernzerhof, "Generalized gradient approximation made simple,” Phys. Rev. Lett. 77, 3865 (1996).

${ }^{60}$ P. E. Blöchl, "Projector augmented-wave method," Phys. Rev. B 50, 17953 (1994).

${ }^{61}$ R. Dronskowski and P. E. Blöchl, "Crystal orbital hamilton populations (COHP): energy-resolved visualization of chemical bonding in solids based on density-functional calculations," J. Phys. Chem. 97, 8617-8624 (1993).

${ }^{62}$ S. Maintz, V. L. Deringer, A. L. Tchougréeff, and R. Dronskowski, "Analytic projection from plane-wave and paw wavefunctions and application to chemical-bonding analysis in solids," J. Comput. Chem. 34, 2557-2567 (2013).

${ }^{63}$ A. Togo, F. Oba, and I. Tanaka, "First-principles calculations of the ferroelastic transition between rutile-type and $\mathrm{CaCl}_{2}$-type $\mathrm{SiO}_{2}$ at high pressures," Phys. Rev. B 78, 134106 (2008).

${ }^{64}$ J. M. Skelton, L. A. Burton, A. J. Jackson, F. Oba, S. C. Parker, and A. Walsh, "Lattice dynamics of the tin sulphides $\mathrm{SnS}_{2}, \mathrm{SnS}$ and $\mathrm{Sn}_{2} \mathrm{~S}_{3}$ : vibrational spectra and thermal transport," Phys. Chem. Chem. Phys. 19, 12452-12465 (2017).

65 J. M. Skelton, "Phonopy-spectroscopy," (2020 (accessed November, 2020)), https://github.com/ JMSkelton/Phonopy-Spectroscopy.

${ }^{66}$ P. Giannozzi, S. Baroni, N. Bonini, M. Calandra, R. Car, C. Cavazzoni, D. Ceresoli, G. L. Chiarotti, M. Cococcioni, I. Dabo, A. Dal Corso, S. de Gironcoli, S. Fabris, G. Fratesi, R. Gebauer, U. Gerstmann, C. Gougoussis, A. Kokalj, M. Lazzeri, L. Martin-Samos, N. Marzari, M. Francesco, R. Mazzarello, S. Paolini, A. Pasquarello, 
L. Paulatto, C. Sbraccia, S. Scandolo, G. Sclauzero, A. P. Seitsonen, A. Smogunov, P. Umari, and R. M. Wentzcovitch, "QUANTUM ESPRESSO: a modular and opensource software project for quantum simulations of materials,” J. Phys.: Condens. Matter 21, 395502 (2009).

${ }^{67}$ M. Methfessel and A. T. Paxton, "High-precision sampling for brillouin-zone integration in metals," Phys. Rev. B 40, 3616 (1989).

${ }^{68}$ G. te Velde, F. M. Bickelhaupt, E. J. Baerends, C. Fonseca Guerra, S. J. A. van Gisbergen, J. G. Snijders, and T. Ziegler, "Chemistry with ADF," J. Comput. Chem. 22, 931-967 (2001).

${ }^{69}$ E. J. Baerends, T. Ziegler, A. J. Atkins, J. Autschbach, D. Bashford, A. Berces, F. M. Bickelhaupt, C. Bo, P. M. Boerritger, L. Cavallo, C. Daul, D. Chong, D. Chulhai, L. Deng, R. M. Dickson, J. M. Dieterich, D. E. Ellis, M. van Faassen, L. Fan, T. H. Fischer, A. Förster, C. Fonseca Guerra, M. Franchini, A. Ghysels, A. Giammona, S. J. A. van Gisbergen, A. Goez, A. W. Götz, J. A. Groeneveld, O. V. Gritsenko, M. Grüning, S. Gusarov, F. E. Harris, P. van den Hoek, Z. Hu, C. R. Jacob, H. Jacobsen, L. Jensen, L. Joubert, J. W. Kaminski, G. van Kessel, C. König, F. Kootstra, A. Kovalenko, M. V. Krykunov, E. van Lenthe, D. A. McCormack, A. Michalak, M. Mitoraj, S. M. Morton, J. Neugebauer, V. P. Nicu, L. Noodleman, V. P. Osinga, S. Patchkovskii, M. Pavanello, C. A. Peeples, P. H. T. Philipsen, D. Post, C. C. Pye, H. Ramanantoanina, P. Ramos, W. Ravenek, M. Reimann, J. I. Rodríguez, P. Ros, R. Rüger, P. R. T. Schipper, D. Schlüns, H. van Schoot, G. Schreckenbach, J. S. Seldenthuis, M. Seth, J. Snijders, M. Solà, M. Stener, M. Swart, D. Swerhone, V. Tognetti, G. te Velde, P. Vernooijs, L. Versluis, L. Visscher, O. Visser, F. Wang, T. A. Wesolowski, E. M. van Wezenbeek, G. Wiesenekker, S. Wolff, T. K. Woo, and Y. A. L., "ADF 2018, SCM, Theoretical Chemistry, Vrije Universiteit, Amsterdam, The Netherlands," (2020 (accessed November, 2020)), http: / / www . scm. com.

${ }^{70}$ E. Van Lenthe and E. J. Baerends, "Optimized slater-type basis sets for the elements 1-118," J. Comput. Chem. 24, 1142-1156 (2003).

${ }^{71}$ W. Sun, S. T. Dacek, S. P. Ong, G. Hautier, A. Jain, W. D. Richards, A. C. Gamst, K. A. Persson, and G. Ceder, "The thermodynamic scale of inorganic crystalline metastability," Sci. Adv. 2, e1600225 (2016).

${ }^{72}$ W. Cui, T. Bi, J. Shi, Y. Li, H. Liu, E. Zurek, and R. J. Hemley, "Route to high- $T_{c}$ superconductivity via $\mathrm{CH}_{4}$ intercalated $\mathrm{H}_{3} \mathrm{~S}$ hydride perovskites," Phys. Rev. B 101, 134504 (2020).

${ }^{73}$ Y. Sun, Y. Tian, B. Jiang, X. Li, H. Li, T. Iitaka, X. Zhong, and Y. Xie, "Computational discovery of a dynamically stable cubic $\mathrm{SH}_{3}$-like high-temperature superconductor at $100 \mathrm{GPa}$ via $\mathrm{CH}_{4}$ intercalation," Phys. Rev. B 101, 174102 (2020).

${ }^{74}$ A. P. Drozdov, M. I. Eremets, and I. A. Troyan, "Superconductivity above $100 \mathrm{~K}$ in $\mathrm{PH}_{3}$ at high pressures," (2015), arXiv:1508.06224 [cond-mat.supr-con].
${ }^{75}$ A. Shamp, T. Terpstra, T. Bi, Z. Falls, P. Avery, and E. Zurek, "Decomposition products of phosphine under pressure: $\mathrm{PH}_{2}$ stable and superconducting?" J. Am. Chem. Soc. 138, 1884-1892 (2016).

${ }^{76}$ J. A. Flores-Livas, M. Amsler, C. Heil, A. Sanna, L. Boeri, G. Profeta, C. Wolverton, S. Goedecker, and E. K. U. Gross, "Superconductivity in metastable phases of phosphorus-hydride compounds under high pressure," Phys. Rev. B 93, 020508(R) (2016).

${ }^{77}$ T. Bi, D. P. Miller, A. Shamp, and E. Zurek, "Superconducting phases of phosphorus hydride under pressure: stabilization by mobile molecular hydrogen," Angew. Chem. Int. Ed. 56, 10192-10195 (2017).

${ }^{78}$ A. K. Mishra, T. Muramatsu, H. Liu, Z. M. Geballe, M. Somayazulu, M. Ahart, M. Baldini, Y. Meng, E. Zurek, and R. J. Hemley, "New calcium hydrides with mixed atomic and molecular hydrogen," J. Phys. Chem. C 122, 19370 19378 (2018).

${ }^{79}$ I. Errea, F. Belli, L. Monacelli, A. Sanna, T. Koretsune, T. Tadano, R. Bianco, M. Calandra, R. Arita, F. Mauri, and J. A. Flores-Livas, "Quantum crystal structure in the 250kelvin superconducting lanthanum hydride," Nature 578, 66-69 (2020).

${ }^{80}$ G. C. Pimentel, "The bonding of trihalide and bifluoride ions by the molecular orbital method," J. Chem. Phys. 19, 446 (1951).

${ }^{81}$ S. J. Grabowski, " $[\mathrm{FHF}]^{-}$- the strongest hydrogen bond under the influence of external interactions," Crystals 6, 3 (2016).

${ }^{82}$ I. Bernal and S. F. Watkins, "Supramolecular fluoride and chloride anions," C. R. Chim. 17, 586-596 (2014).

${ }^{83}$ J. L. Manson, H. I. Southerland, B. Twamley, R. Rai, and L. Musfeldt, "Bis(di-2-pyridyl-methanediol)copper(II) dihydrogentrifluoride: a structural and spectroscopic study of the $\mathrm{H}_{2} \mathrm{~F}_{3}^{-}$anion in a complex salt," Dalton Trans. , 56555657 (2007).

${ }^{84}$ S. I. Troyanov, I. V. Morozov, and E. Kemnitz, "Crystal structure of cesium dihydrotrifluoride, $\mathrm{CsH}_{2} \mathrm{~F}_{3}$. refinement of the crystal structures of $\mathrm{NMe}_{4} \mathrm{HF}_{2}$ and $\mathrm{NMe}_{4} \mathrm{H}_{2} \mathrm{~F}_{3}$," $\mathrm{Z}$. Anorg. Allg. Chem. 631, 1651-1654 (2005).

${ }^{85} \mathrm{D}$. Mootz and D. Boenigk, "Fluorides and fluoro acids. 12. complex-anion homology and isomerism in the crystal structures of two potassium poly(hydrogen fluorides), KF.5HF and KF.3HF," J. Am. Chem. Soc. 108, 6634-6636 (1986).

${ }^{86}$ D. Wiechert, D. Mootz, R. Franz, and G. Siegemund, "Amine-poly(hydrogen fluoride) solid complexes: new studies of formation, crystal structures, and $\mathrm{H}_{n-1} \mathrm{~F}_{n}^{-}$ion diversity," Chem. Eur. J. 4, 1043-1047 (1998).

${ }^{87}$ T. Oka, "Interstellar $\mathrm{H}_{3}^{+}$," Proc. Natl. Acad. Sci. U.S.A. 103, 12235-12242 (2006).

${ }^{88}$ Z. Wang, H. Wang, J. S. Tse, T. Iitaka, and Y. Ma, "Stabilization of $\mathrm{H}_{3}^{+}$in the high pressure crystalline structure of $\mathrm{H}_{n} \mathrm{Cl}(n=2-7)$," Chem. Sci. 6, 522-526 (2015).

${ }^{89}$ Q. Zeng, S. Yu, D. Li, A. R. Oganov, and G. Frapper, "Emergence of novel hydrogen chlorides under high pressure,” Phys. Chem. Chem. Phys. 19, 8236-8242 (2017). 
${ }^{90}$ D. Duan, X. Huang, F. Tian, Y. Liu, D. Li, H. Yu, B. Liu, W. Tian, and T. Cui, "Predicted formation of $\mathrm{H}_{3}^{+}$in solid halogen polyhydrides at high pressures," J. Phys. Chem. A 119, 11059-11065 (2015).

${ }^{91}$ J. A. Ibers, "Refinement of peterson and levy's neutron diffraction data on $\mathrm{KHF}_{2}$," J. Chem. Phys. 40, 402 (1964).

${ }^{92}$ B. L. McGaw and J. A. Ibers, "Nature of the hydrogen bond in sodium acid fluoride," J. Chem. Phys. 39, 2677 (1963).

${ }^{93}$ M. W. Johnson, E. Sándor, and E. Arzi, "The crystal structure of deuterium fluoride," Acta Cryst. B31, 1998-2003 (1975).

${ }^{94}$ D. A. Pinnick and R. C. Katz, A. I. ad Hanson, "Pressure effects on raman scattering from solid HF and DF," Phys. Rev. B 39, 8677 (1989).

${ }^{95} \mathrm{Z}$. Wang, H. Cui, J. Hou, and X. Dong, "Can $\mathrm{HHe}^{+}$ exist at high pressure: Exploration of high pressure induced HF-He compounds," Geosci. Front. (2020), https://doi.org/10.1016/j.gsf.2020.03.010.
${ }^{96}$ M. Miao, Y. Sun, E. Zurek, and H. Lin, "Chemistry under high pressure," Nat. Rev. Chem. 508-527, 4 (2020).

${ }^{97}$ B. M. Riggleman and H. G. Drickamer, "Approach to the metallic state as obtained from optical and electrical measurements," J. Chem. Phys. 38, 2721 (1963).

${ }^{98}$ T. Kenichi, S. Kyoko, F. Hiroshi, and O. Mitsuko, "Modulated structure of solid iodine during its molecular dissociation under high pressure," Nature 423, 971-974 (2003).

${ }^{99}$ K. Shimizu, T. Yamauchi, N. Tamitani, N. Takeshita, M. Ishizuka, K. Amaya, and S. Endo, "The pressureinduced superconductivity of iodine," J. Supercond. 7, 921-924 (1994).

${ }^{100}$ P. B. Allen and R. C. Dynes, "Transition temperature of strong-coupled superconductors reanalyzed," Phys. Rev. B 12, 905 (1975).

${ }^{101}$ Http://hdl.handle.net/10477/79221, July 05, 2020. 